\title{
Power Index of the Inspiratory Flow Signal as a Predictor of Weaning in Intensive Care Units*
}

\author{
Javier A. Chaparro ${ }^{1}$, Beatriz F. Giraldo ${ }^{2}$, Senior Member, IEEE
}

\begin{abstract}
Disconnection from mechanical ventilation, called the weaning process, is an additional difficulty in the management of patients in intensive care units (ICU). Unnecessary delays in the discontinuation process and a weaning trial that is undertaken too early are undesirable. In this study, we propose an extubation index based on the power of the respiratory flow signal $\left(P_{i}\right)$. A total of 132 patients on weaning trials were studied: 94 patients with successful trials (group S) and 38 patients who failed to maintain spontaneous breathing and were reconnected (group $F$ ). The respiratory flow signals were processed considering the following three stages: a) zero crossing detection of the inspiratory phase, b) inflection point detection of the flow curve during the inspiratory phase, and c) calculation of the signal power on the time instant indicated by the inflection point. The zero crossing detection was performed using an algorithm based on thresholds. The inflection points were marked considering the zero crossing of the second derivative. Finally, the inspiratory power was calculated from the energy contained over the finite time interval (between the instant of zero crossing and the inflection point). The performance of this parameter was evaluated using the following classifiers: logistic regression, linear discriminant analysis, the classification and regression tree, Naive Bayes, and the support vector machine. The best results were obtained using the Bayesian classifier, which had an accuracy, sensitivity and specificity of $87 \%, 90 \%$ and $81 \%$ respectively.
\end{abstract}

\section{INTRODUCTION}

Mechanical ventilation is the principal medical procedure used in intensive care units to assist patients with acute respiratory failure. Around $50 \%$ of patients in intensive care units require mechanical ventilation [1]-[3]. This process can be applied in an invasive or noninvasive form.

Weaning is the process of transferring the work of breathing from the ventilator back to the patient, and is still one of the most challenging problems in clinical practice [4], [5]. Withdrawal of mechanical ventilation should be performed as soon as patients can breathe on their own. Both unnecessary delay and premature weaning may have adverse

*This work was supported in part by the Escuela Colombiana de Ingeniería Julio Garavito, Bogotá Colombia, and the Spanish Government's Ministerio de Economía y Competitividad under grant TEC2010-21703C03-01.

${ }^{1}$ J.A. Chaparro is with Escuela Colombiana de Ingeniería. Programa de Ingeniería Electrónica. Grupo de investigación Ecitronica. Bogotá, Colombia. (e-mail: javier.chaparro@escuelaing.edu.co).

${ }^{2}$ B.F. Giraldo is with Dept. ESAII, Escola Universitaria de Enginyeria Tècnica Industrial de Barcelona (EUETIB), Universitat Politècnica de Catalunya (UPC), Institut de Bioingenyeria de Catalunya (IBEC), and CIBER de Bioingeniería, Biomateriales y Nanomedicina (CIBER-BBN). c/. Pau Gargallo 5, 08028, Barcelona, Spain (e-mail:beatriz.giraldo@upc.edu ). effects on patient outcome, prolonging mechanical ventilation and length of stay in the intensive care unit [6], [7]. When mechanical ventilation is discontinued, up to $25 \%$ of patients have respiratory distress severe enough to require reinstitution of ventilator support [8].

Several studies have been performed to identify physiological variables that are useful indicators for undertaking a weaning trial [9]-[11], strategies to reduce the duration of mechanical ventilation and risk factors of unsuccessful discontinuation [12], and whether mortality in mechanically ventilated patients has changed over time [13].

In addition, criteria like minute volume, maximum inspiratory pressure, tidal volume, rapid shallow breathing, and CROP (compliance, resistance, oxygenation pressure) have been analyzed as indicators to determine whether a patient can come off the ventilator. Most of these tests are sensitive, but not specific [14], [15]. Therefore, the process of discontinuing mechanical ventilation is still one of the most challenging problems in intensive care.

In this study, we propose a new index to characterize the respiratory dynamic of patients on an intensive care unit undergoing mechanical ventilation and in the weaning process. This index is based on the signal power of the respiratory flow $\left(P_{i}\right)$, obtained from the inspiratory phase. For each inspiratory phase: a) the zero crossing time $\left(t_{z}\right)$ is detected, and validated according to an energy threshold $\left(E_{z}\right)$; b) the time of the maximum inflection in the inspiratory phase $\left(t_{i}\right)$ is calculated; and c) the cumulative energy $\left(E_{i}\right)$ between $t_{z}$ and $t_{i}$ is calculated. Finally, $P_{i}$ is defined as the ratio between $E_{i}$ and its time interval $\left(t_{i}-t_{z}\right)$.

This new $P_{i}$ index was used to identify patients with successful spontaneous breathing trials and patients with unsuccessful trials. Classifiers such as logistic regression, linear discriminant analysis (LDA), and the classification and regression tree (CART), the Naive-Bayes technique, and a support vector machine (SVM) were used to determine the best results. The aim of this study was to assess the contribution of this new index to identifying patients who could successfully wean. The results were evaluated in terms of accuracy, sensitivity and specificity.

\section{METHODOLOGY}

\section{A. Datasets}

Respiratory flow signals were recorded for 132 patients on weaning trials from mechanical ventilation, at Santa Creu i Sant Pau Hospital, Barcelona, Spain, and Getafe Hospital, Getafe, Spain. All subjects were studied according to a protocol approved by the local ethics committee. The records 
were obtained with a pneumotachograph, connected to an endotracheal tube, and consisting of a Datex-Ohmeda monitor (GE Healthcare, Milwaukee, WI, USA) and a Validyne MP45-1-871 Variable-reluctance Transducer (Validyne Corp., Northridge, CA, USA). The signals were recorded for $30 \mathrm{~min}$, at a $250-\mathrm{Hz}$ sampling rate and 12-bit resolution.

Patients undergoing a spontaneous breathing trial were disconnected from the ventilator and left to breathe through an endotracheal tube for $30 \mathrm{~min}$. If the patients maintained spontaneous breathing with normality they were extubated, otherwise, they were reconnected. When patients still maintained spontaneous breathing after $48 \mathrm{~h}$, the weaning trial process was considered successful; otherwise, the patients were reintubated. According to the clinical criteria, the patients were classified into two groups: group S, 94 patients (61 male, 33 female, aged $65 \pm 17$ years) who weaned successfully; and group F, 38 patients ( 24 male, 15 female, aged $67 \pm 15$ years) who failed to maintain spontaneous breathing.

\section{B. Characterization of Respiratory Flow Signals}

In order to obtain the power signal of the respiratory flow for each inspiratory cycle, the next parameters were extracted: the time value to zero crossing named $t_{z}$, the time value of the maximal inflection named $t_{i}$, and the cumulative energy of the signal between these two time intervals named $E_{i}$. Finally, the $P_{i}$ index was calculated.

- Zero crossing detection. The respiratory flow signal was analyzed by computing the difference between two consecutive points. Zero crossing is the point where the sign of these differences changes. The inspiratory cycles are detected when the changes are from negative to positive values. To define a correct detection, the energy for the following points of the signal is calculated by

$$
E_{a}(t)=\sum_{t_{z}}^{t} x(t)^{2}
$$

where $x(t)$ is the respiratory flow signal, and $E_{a}(t)$ represents the energy accumulated since the last zero crossing in $t_{z}$, up to $t$. The threshold energy to consider a valid zero point was fixed at $0.1(\mathrm{~L} / \mathrm{s})^{2}$. A correct zero crossing, called $t_{z}$, was defined when $E_{a}(t)$ was above this threshold. Figures 1(a) and (c) show an example of a zero-crossing detection.

- Inflection point detection. The next step was to detect an inflection point after the zero crossing. This point is determined by changes in the curvature of the signal, and is obtained using the second derivative (Figures $1[\mathrm{~b}]$ ). The new point is called $t_{i}$.

- Power index calculation. The power index $\left(P_{i}\right)$ obtained from the energy contained over a finite time interval $\left(T_{i z}=t_{z}-t_{i}\right)$ is defined as

$$
P_{i}=\frac{1}{T_{i z}} \sum_{t_{z}}^{t_{i}} x(t)^{2}
$$
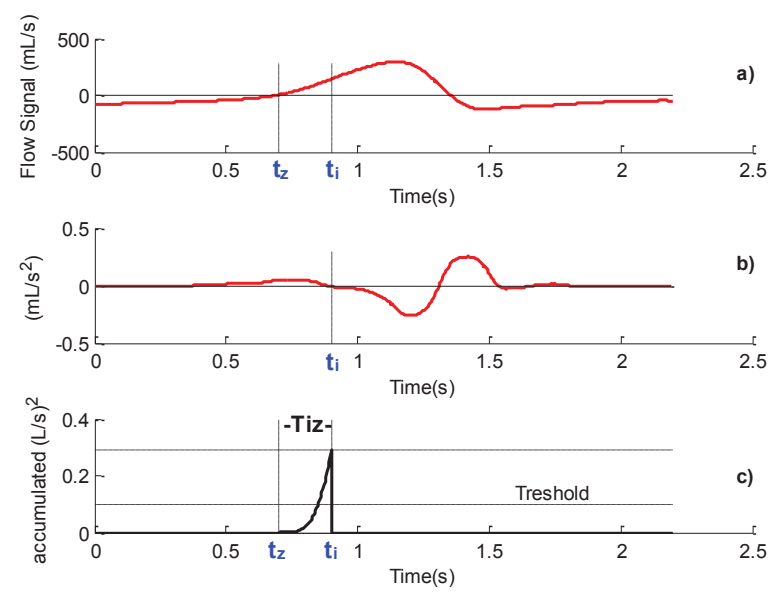

Figure 1. A respiratory cycle with a) zero crossing and inflection point marks, b) the second derivative to define this inflection point, and c) the signal energy accumulated between $t_{z}$ and $t_{i}$.

\section{Classification Methods}

In order to assess the Pi index for classifying successful and unsuccessful patients on weaning trials from mechanical ventilation, the following methods were applied. The leaveone-out procedure was used to validate the results.

- Logistic regression (LR) is an approach to prediction, such as ordinary least squares regression, from a categorical outcome through one or more predictor parameters [16]. The model is given by

$$
P=\frac{1}{1+e^{-\left(\alpha_{0}+\alpha_{1} x_{i}\right)}}
$$

where $P$ is the occurrence probability of an event of the $\mathrm{x}_{i}$ index, and $\alpha_{l}$ is the weight of the parameter. The coefficients are estimated using the maximum likelihood method.

- Linear discriminant analysis (LDA) is used to find a linear combination of features that characterize two or more targets. This method maximizes the variance ratio between and within-class in any data set, guaranteeing maximal separability [17]. It has been defined as

$$
Y=\mu_{0}+\mu_{1} x_{i}
$$

where $x_{i}$ and $\mu_{0}$ are the independent parameters and independent term, respectively, and $\mu_{l}$ is the discriminant function coefficient.

- Decision tree uses a predictive model to try to best predict the probability of an outcome. The classification and regression tree (CART) analysis covers both functions. This method usually works top-down, by choosing a variable at each step that best splits the set of items. The inputs are represented by a set of predictor attributes [18], [19]. 
- Naïve Bayes Classifier is a probabilistic classifier based on Bayes' theorem applied to supervised classification with discrete data. There are different types, depending on the relationship of dependence between the predictor variables [19]. The classification model is based on the independent predictor variables $X_{i}$ (symptoms variables), given the class variable $C$ (diagnostic variable). The most probable a posteriori diagnosis $c^{*}$, known of symptoms $\boldsymbol{X}=\left(X_{1}, \ldots, X_{n}\right)$, can be considered as the state $C$ with the highest a posteriori probability, and is given by

$$
c^{*}=\underset{c}{\arg \max } p\left(C=c \mid X_{1}, \cdots, X_{n}\right)
$$

- Super vector machines (SVM) are based on transforming data into a higher dimensional space to convert a complex classification problem into a simpler one that can be solved by a linear discriminant function, known as a hyperplane, and defined by [20], [21]

$$
f(x)=\omega z+b=\sum_{i}^{L} \propto_{i} y_{i} K\left(x_{i}, y_{i}\right)+b
$$

where $\alpha_{i}$ and $b$ are determined to solve a large-scale quadratic programming problem, for which efficient algorithms exist that guarantee global optimum values.

\section{Performance evaluation measures}

The differences between groups were tested by the Mann-Whitney $U$ test. The quality of the results was analyzed in terms of accuracy $(A c c)$, sensitivity $\left(S_{n}\right)$ and specificity $\left(S_{p}\right)$ in each case. These measures are built from a confusion matrix, which shows a binary classification where (the values) $t_{p}$ are true positive, $f_{p}$ false positive, $t_{n}$ true negative, and $f_{n}$ false negative [22]. These measures are defined as

$$
\begin{gathered}
A c c=\frac{t_{p}+t_{n}}{t_{p}+f_{p}+t_{n}+f_{n}} ; \\
S_{n}=\frac{t_{p}}{t_{p}+f_{n}} ; \quad S_{p}=\frac{t_{n}}{f_{p}+t_{n}}
\end{gathered}
$$

\section{RESULTS}

Firstly, the respiratory flow signals were preprocessed. A smoothing filter of 20 samples was applied. Secondly, the times of zero crossing and the inflection point of the inspiratory cycles were calculated. Figure 2 shows an example of the marks of these times in some respiratory cycles, and the area under the curve that was used to calculate the power. Thirdly, the $P_{i}$ index was calculated for each inspiratory cycle. Figure 3 presents the power index $P_{i}$ obtained for successful and failed patient groups.

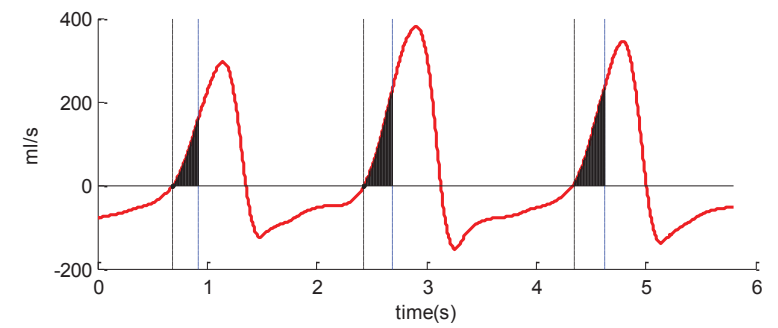

Figure 2. Respiratory flow signal with the time zero crossing, the time of the inflection point, and the area under the curve where the energy $E_{i}$ is calculated.

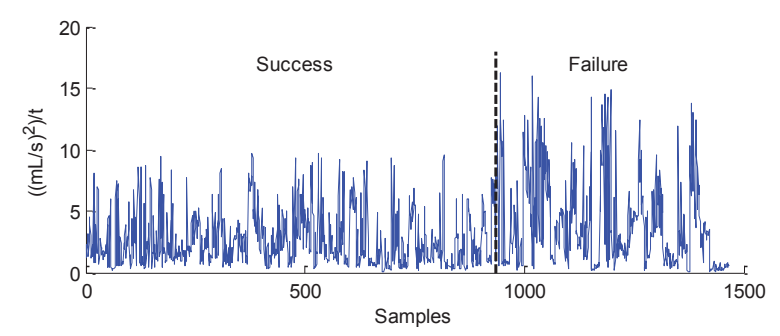

Figure 3. Power index $P_{i}$ obtained from successful and failed patient groups.

Considering a signal length of $1 \mathrm{~min}$, the mean and standard deviation of the $P_{i}$ index was calculated. According to the results, this index present statistically significant differences ( $p$-value $<0.0001)$ in comparisons of the successful $(2.99 \pm 2.68)$ and failed groups $(3.76 \pm 3.59)$. The performance of the parameter was evaluated using the following classifiers: logistic regression (LR), linear discriminant analysis (LDA), the classification and regression tree (CART), Naive Bayes (NB), and the support vector machine (SVM). Table I presents the results obtained with these classifiers, in terms of accuracy, sensitivity and specificity, as the mean and standard deviation of the signal length of $1 \mathrm{~min}$.

TABLE I. MEAN AND STANDARD DEVIATION OF ACCURACY (ACC), SENSITIVITY $\left(\mathrm{S}_{\mathrm{N}}\right)$ AND SPECIFICITY $\left(\mathrm{S}_{\mathrm{p}}\right)$ OBTAINED WITH LR, LDA, CART, NB AND SVM CLASSIFFIERS USING A SIGNAL LENGTH OF 1 MIN.

\begin{tabular}{cccccc}
\hline$P_{i}$ & $\boldsymbol{L R}$ & $\boldsymbol{L D} \boldsymbol{C}$ & $\boldsymbol{C A R T}$ & $\boldsymbol{N B}$ & $\boldsymbol{S W M}$ \\
\hline Acc & $0.59 \pm 0.07$ & $0.61 \pm 0.01$ & $0.81 \pm 0.03$ & $0.87 \pm 0.01$ & $0.64 \pm 0.01$ \\
$\mathrm{~S}_{\mathrm{n}}$ & $0.85 \pm 0.19$ & $0.84 \pm 0.02$ & $0.90 \pm 0.01$ & $0.90 \pm 0.01$ & $0.95 \pm 0.01$ \\
$\mathrm{~S}_{\mathrm{p}}$ & $0.10 \pm 110$ & $0.26 \pm 0.01$ & $0.66 \pm 0.06$ & $0.81 \pm 0.02$ & $0.12 \pm 0.02$ \\
\hline
\end{tabular}

The CART and NB classifiers had the best classification rate at $81 \%$ and $87 \%$, respectively. All classifiers had high specificity values, particularly the CART, NB and SVM, but with the best sensitivity result was obtained with the NB classifier.

The performance of the $P_{i}$ was also analyzed with respect to signal length for ranges from 0.5 and 1 to 5 min. Figure 4 presents the accuracy evaluated in these signal lengths. The results show that with a length of 1 min the naive Bayes classifier performed better than the other classifiers. 


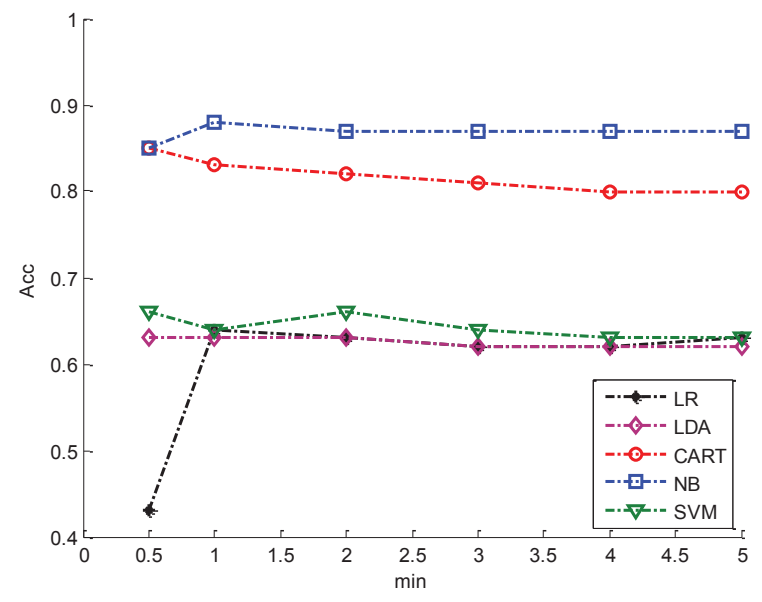

Figure 4. Accuracy (Acc) as a function of signal length, using the classifiers LR, LDA, CART, NB and SVM.

\section{CONCLUSIONS}

In this study, we have proposed a new $P_{i}$ index calculated from the power for each inspiratory cycle. The procedure involves the following steps: define the interval time to calculate the power of the signal, calculate the power index, and use this index to classify patients who have succeeded in the extubation process and patients who have failed.

The interval times used to calculate the energy information of the signals were obtained from the time of zero crossing up to the inflection point of the inspiratory cycle using the second derivative of the signal as a criterion. The threshold to determine a correct zero point was fixed at $0.1(\mathrm{~L} / \mathrm{s})^{2}$.

The power index calculated from the energy of the signal showed statistically significance differences between the two groups of patients, and was higher in the failed group than in the successful group.

With signal lengths of $1 \mathrm{~min}$, the best classification was obtained using the Naive Bayes classifier (87\%), which had the best relation between sensitivity $(90 \%)$ and specificity (81\%). According to these results, 1-min signals could provide relevant information about a patient's condition. Thus, an advantage of this new index is its prognostic value using short respiratory flow signals. Although promising, the significance of the results needs to be further established on a larger set.

\section{ACKNOWLEDGMENT}

The authors would like to thank the staff of the Intensive Care Departments at Santa Creu i Sant Pau Hospital, Barcelona, and at Hospital Universitario de Getafe, Getafe, Spain, for their collaboration with the signal database

\section{REFERENCES}

[1] M. J.F. and J. Kress, "Weaning patients from the ventilator," The new England Journal of Medicine, vol. 367, pp. 2233-9, 2012.
[2] J-M. Boles, J. Bion, A. Connors, M. Herridge, B. Marsh, C. Melot, R. Pearl, H. Silverman, M. Stanchina, A. Vieillard-Baron, T. Welte 11. "Weaning from mechanical ventilation". European Respiratory Journal. No. 29: 1033-1056. 2007.

[3] Tobin, M.J., M.J. Mador, S.M. Guenter, R.F. Lodato, M.A. Sackner, "Variability of resting respiratory center drive and timing in healthy subjects". J. Appl. Physiol., No. 65, pp. 309-317. 1998.

[4] Blackwood, B., Alderdice, F., Burns, K., Cardwell, C., Lavery, G., and O'Halloran, P. (2011). Use of weaning protocols for reducing duration of mechanical ventilation in critically ill adult patients: Cochrane systematic review and meta-analysis. BMJ, 342 (Jan13 2):c7237-c7237.

[5] Burns, K., Meade, M., Lessard, MR Hand, L., Zhou, Q., Keenan, S., and Lellouche, F. (2013). Wean earlier and automatically with new technology (the wean study). a multicenter, pilot randomized controlled trial. Am J Respir Crit Care Med, 187(11):12031211.

[6] H.R. Hemant, J. Chacko, M.K. Singh, "Weaning from mechanical ventilation- current evidence". Indian Journal of Anaesth; No. 50(6), pp 435-438. 2006.

[7] Casaseca de la Higuera, P., Martín Fernandez, M., \& Arbeloa López, C. Weaning from mechanical ventilation: a retrospective analysis leading to a multimodal perspective. IEEE Transaction on biomedical engineering, No. 57(7), pp 1330-1345. 2006.

[8] M.J. Tobin, "Advances in mechanical ventilation", N. Engl. J. Med.,Vol. 344, N. 26, pp. 1986-1996, 2001.

[9] Santos Lima, E. J. (2013). Respiratory Rate as a Predictor of Weaning Failure from Mechanical Ventilation. Brazilian Journal of Anesthesiology (English Edition), 63(1):1-6.

[10] Stawicki, S. P. (2007). Mechanical ventilation: Weaning and extubation. OPUS 12 Scientist, 1(2):13-16.

[11] J. Chaparro, B. Giraldo, P. Caminal, S. Benito. "Performance of Respiratory Pattern Parameters in Classifiers for Predict Weaning Process”. Engineering in Medicine and Biology Society, IEMBS '12. 34th Annual International Conference of the IEEE. 2012.

[12] McConville, J. F. and Kress, J. P. (2012). Weaning Patients from the Ventilator. New England Journal of Medicine, 367(23):2233-2239.

[13] Esteban, A., Frutos-Vivar, F., Muriel, A., Ferguson, N. D., Penuelas, O., et al. (2013). Evolution of mortality over time in patients receiving mechanical ventilation. Am J Respir Crit Care Med, 188(2): 220230.

[14] Jiin-Chyr Hsu, Yung-Fu Chen, Hsuan-Hung Lin, Chi-Hsiang Li and Xiaoyi Jiang, "Construction of Prediction Module for Successful Ventilator Weaning", New Trends in Applied Artificial Intelligence, pp. 766-775, 2007.

[15] Chao DC and Scheinhorn DJ, "Determining the Best Threshold of Rapid Shallow Breathing Index in a Therapist-Implemented PatientSpecific Weaning Protocol", Respir Care 2007; 52(2):159 -165.

[16] H.Tinsley and S. Brown, "Handbook of applied multivariate statistics and mathematical modeling," Academic Press, 2000.

[17] Huberty C., "Applied Discriminant Analysis, Whiley Series in Probability and Mathematical Statistics", Editorial Jhon Wiley \& Sons Inc., 1994.

[18] C. Kingsford and S.L Salzberg, "What are decision trees?".Nat Biotechnol, Vol. 26, No. 9, pp. 1011-1013, 2008.

[19] U. Fayyad, G. Piatetsky-Shapiro, and P. Smyth, "From Data Mining to Knowledge Discovery in Databases". American Association for Artificial Intelligence, pp. 0738-4602, 1996.

[20] Steinwart I., Chrismann A., "Super Vector Machine, Information Science and Statistics", Editorial Springer. 2008.

[21] A. Garde, R. Schroeder, A. Voss, P. Caminal, S. Benito and B.F. Giraldo, "Patients on weaning trials classified with support vector machines", Physiol. Meas. 31, pp. 979-993, 2010.

[22] M. Sokolova, N. Japkowicz, and S. Szpakowicz, "Beyond Accuracy,F-Score and ROC: A Family of Discriminant Measures forPerformance Evaluation," Advances in Artificial Intelligence pp.1015-1021, 2006. 